\title{
Distributed Data Management Service for VPH Applications
}

\author{
Spiros Koulouzis, Adam Belloum, Marian Bubak, Pablo Lamata, Daniel Nolte, Dmitry Vasyunin, \\ Cees de Laat
}

\begin{abstract}
For many medical applications it is challenging to transparently access large datasets, often hosted across different domains on heterogeneous infrastructures. Homogenizing the infrastructure to simplify data access is unrealistic, therefore, it is important to elaborate a distributed storage which doesn't introduce added complexity. The main objective of this research is to investigate a solution that flexibly federates data on clouds or grids without any changes to the existing infrastructure and to be able to scale with the increasing data demand. Unlike existing approaches, ours isnt bound to a specific technology and doesn't require from administrators, users or application developers additional effort to federate and use the storage infrastructure. The elaborated system is implemented on the VPH-Share infostructure. As an example, we present a cardiac anatomy analysis workflow which uses the proposed solution to support a novel diagnostic method to characterize the shape of the heart.
\end{abstract}

Index Terms—data federation; data sharing; cloud storage; VPH applications; cardiac anatomy

\section{INTRODUCTION}

Virtual Physical Human (VPH) ${ }^{1}$ is a methodology and a framework aiming at the collaborative investigation of the human body. VPH is an important step towards personalized medicine. Advances in medical technology have created large medical records. The challenge of the VPH community is to access and share these records effectively while complying with ethical and legal restrictions. Medical data combined with novel diagnostic methods can improve the accuracy and personalization of diagnoses and reduce the cost and time of healthcare. Therefore, researchers and applications need access large datasets that are geographically distributed across multiple organizations. A number of VPH applications are designed to access data from local disk, which will not work if the data is stored in some remote storage system. Fragmentation of datasets also complicates data discovery and makes it time-consuming for users who often have limited knowledge of IT solutions.

Data storage federation can address these problems. It aims at virtualizing storage resources located at different independent institutions and present them through a

- S. Koulouzis, A. Belloum, D. Vasyunin, C. de Laat are with Informatics Institute, University of Amsterdam. E-mails: \{S.Koulouzis,A.S.Z.Belloum, Dvasunin,C.T.A.M.deLaat\}@uva.nl

- P. Lamata,D. Nolte are with the Department of Biomedical Engineering, King's College London, UK. E-mails: \{pablo.lamata,daniel.nolte\}@kcl.ac.uk

- M. Bubak is with the AGH University of Science and Technology Krakow, Poland and the Informatics Institute, University of Amsterdam. E-mails: M.T.Bubak@uva.nl,bubak@agh.edu.pl.

This work was partly funded by the European Commission (FP7ICT-269978:VPH-Share). and COMMIT(http://www.commit-nl.nl/) projects. P.Lamata holds a holds a Sir Henry Dale Fellowship jointly funded by the Wellcome Trust and the Royal Society (099973/Z/12/Z). The authors would like to acknowledge the work of the whole VPH-Share consortium.

1. http://www.vph-institute.org/ unified view. Our approach to data storage federation is to aggregate a pool of independent and heterogeneous resources, including storage clouds and data grids and present them via WebDAV ${ }^{2}$ offering a simple file system abstraction. This makes the development and deployment of applications simpler and improves their reliability. Experience has shown that many execution failures in distributed environments are attributed to missing files [1]. Our solution also helps to use available storage space efficiently, using a copy-on-write strategy. When separate logical data use identical copies of the same physical data, it is not necessary to create separate copies as they can all be given pointers to the same data.

The main objective of the research presented in this paper is to elaborate an architecture that can flexibly federate clouds, grids and other storage technologies without any changes to the infrastructure. This research presents a distributed large-scale virtual file system that can take advantage of all available technologies providing a global shared view of heterogeneous and distributed storage resources. The result is an easy-to-use, intuitive service that hides the complexity of federation using a standardized access protocol.

The elaborated system is used by the VPH-Share project community. As an example we present a cardiac anatomy analysis workflow. This workflow is a novel methodological approach to the analysis of the cardiac anatomy. The vision of this workflow is to provide a cloud-enabled diagnostic method to characterize the shape and the material properties of the heart.

2. Web Distributed Authoring and Versioning protocol is an extension of HTTP allowing remote file transfers. 


\section{Related Work}

Many proposals attempt to realize storage federation and unify data access. These proposals are often tightly coupled with a storage infrastructure or technology which minimizes flexibility and makes it difficult to adapt to hardware and software changes. Existing solutions often implement "ad-hoc" interfaces to provide access to users and applications. These designs reduce interoperability and in most cases are specific for grid or cloud environments.

Globus Online [2] is a data transport service that federates GridFTP [3] resources for moving large quantities of data across geographically distributed storage systems. Although Globus online is able to transfer huge amounts of data, it is limited to coordinating transfers between GridFTP servers.

The approach described in [4] implements redundant storage techniques on cloud systems to allow customers to avoid vendor lock-in ${ }^{3}$, reduce the cost of switching providers and to tolerate provider outages or failures. This approach is limited to the Amazon S3 storage.

PhysiomeSpace is a solution developed with the VPH community in mind [5]. PhysiomeSpace puts emphasis on sharing and managing medical images and supports almost any type of biomedical data. However, it uses a custom developed client to make the data available to users making it less flexible and requiring more effort to maintain compatibility of the custom client with the service. Additionally, the proposed solution is limited to use Storage Resource Broker (SRB) [6].

VeilFS is a system that attempts to unify access to files stored at heterogeneous data storage systems that belong to geographically distributed organizations [7]. This approach is designed to only use local storage. VeilFS also uses custom access protocols forcing users to adopt a dedicated client implemented only for VeilFS.

Unlike existing proposals, our approach takes advantage of all available technologies to offer a large scale collaborative storage environment to the VPH community. Our architecture is able to federate multiple, heterogeneous and independent storage resources and present them as a unified storage space.

\section{System Architecture \& Its Compo- NENT}

The Large OBject Cloud Data storagE fedeRation (LOBCDER) is a data management system that federates storage resources. It is a part of the Data and Compute Cloud Platform ${ }^{4}$ of the VPH-Share project [8]. Its function is to provide a large and scalable file storage that mimics a local file system. In [9] we describe a 2-tier architecture of LOBCDER which is a centralized solution to federated storage. This architecture will not

3. Vendor lock-in occurs when a user cannot migrate data from a cloud storage offering, because the price of downloading all the data at once, exceeds the benefit of using a cheaper vendor

4. LOBCDER is available via https://portal.vph-share.eu/data/. scale when the number of requests increases. In this paper we propose a distributed architecture. The distributed LOBCDER is composed of three components, the Read/Write (RW) master, the Read (R) master and the workers shown in Figure 1.

The LOBCDER master is divided into three layers: the frontend, the resources and the backend. The frontend is a WebDAV interface offering resource locking to maintain data consistency. The resource layer holds a logical representation of the physical data and manages the physical storage space. The logical representation (metadata) is stored in a database and together with the master may be replicated across several machines to avoid single point of failure. The synchronization of the logical representation is achieved by allowing only one the RW master to perform all uploads and updates while downloads can be served the $\mathrm{R}$ master. The backend enables the system to uniformly access physical storage resources. Currently LOBCDER is able to use a wide range of storage systems like Local File System, SFTP, WebDAV, GridFTP, SRM, LFC iRODS, OpenStack-Swift, vCloud, AWS S3.

The LOBCDER worker is a stateless service composed of two layers: the frontend and backend. The frontend processes HTTP requests to download files. For each request, the worker gets a reference to the data from the master and uses it to relay data stored in the backend to the client. The number of LOBCDER workers can be increased or decreased following the number of incoming data transfer requests.

To download a file from LOBCDER (see Figure 1) the RW master authenticates and authorizes the user (step 1), and selects a worker to transfer the data based on load-balancing algorithms described in Section 5 . When a worker is selected, the RW master generates a one-time password (OTP) for the user. The user is redirected to the selected worker to proceed with the transfer (step 2). When the user connects to the worker, it provides the OTP. If the user is authenticated the worker gets information about the physical data location from the master (step 4). The worker selects the most suitable storage resource to relay the data to the user. The selection of the storage resources takes into account the performance of the resource gathered from previous transfers (step 6). Finally, the worker relays the data to the user (step 7,8).

All communications between the LOBCDER master and workers are over a secured communication channel (SSL) to protect the OTP. As an extra measure of security, the master restricts access to information to registered works.

To increase availability, reliability and reduce access latency LOBCDER replicates data on available storage resources based on the demand on each file. The default strategy for an uploaded file is to copy it to the most reliable storage resource first. The reliability of each resource is calculated based on the successful downloads. Frequently requested files are replicated to storage 


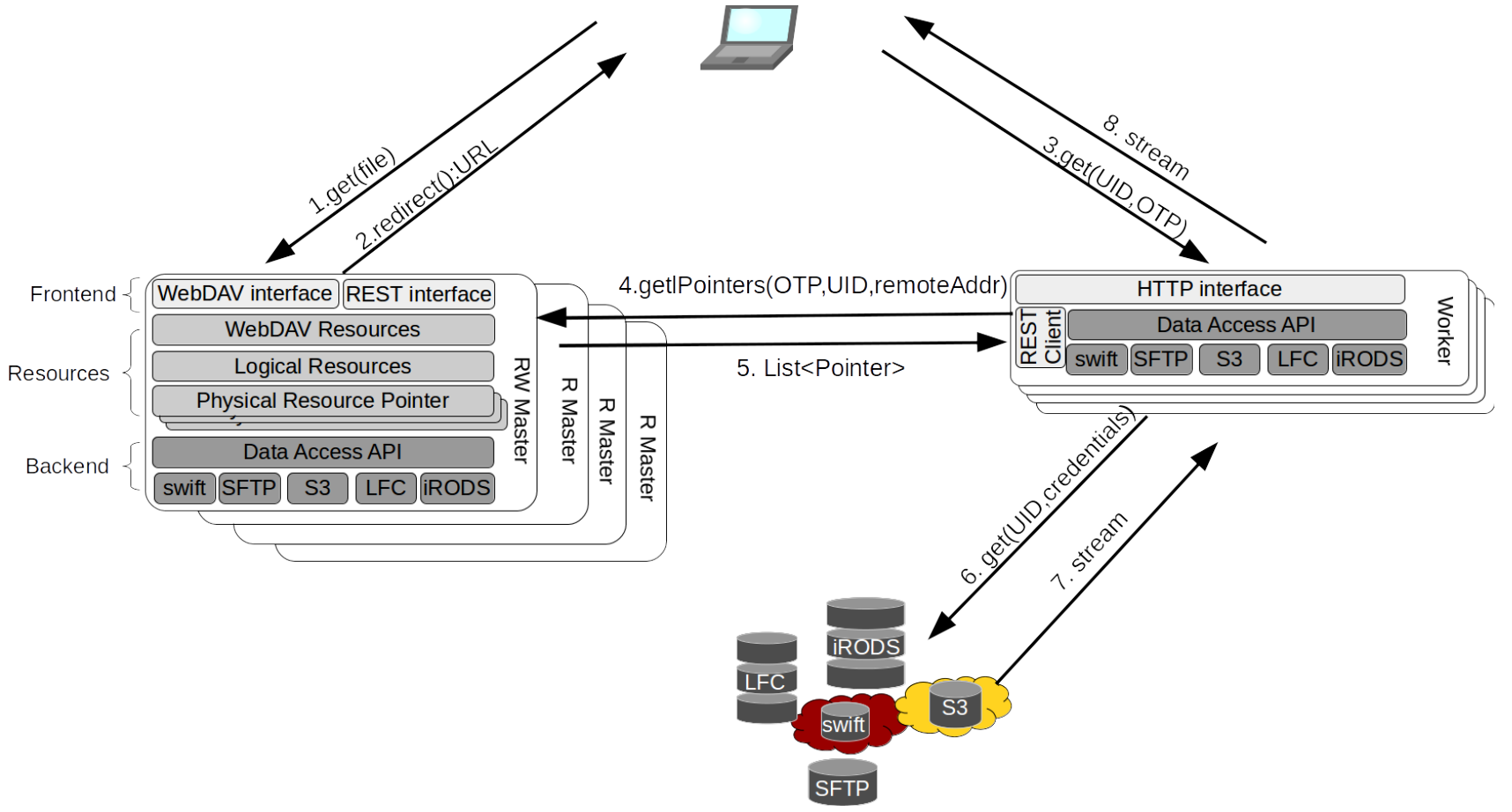

Fig. 1. LOBCDER's architecture. The masters hold the logical representation of the file system. The workers serve GET requests which are redirected from the masters.

resources that are closer to the requests. Additionally, while a worker sends a file to a user and has enough space on its local disk, the file is cached locally.

\section{Consistency, Avallability, Partition}

The Consistency, Availability, Partition (CAP) theorem, states that any networked shared-data system can satisfy two of the three properties: consistency, availability, and partition tolerance [10]. LOBCDER is used as a shared file system therefore it is important to maintain consistency towards clients. The Analysis of the VPH-share data access patterns shows that availability is not hard to maintain. A typical scenario for making a dataset available within VPH-Share starts with a data owner uploading a dataset into LOBCDER. When other users want to use this dataset they send a request to the data owner who will grant access to the dataset if certain requirements are satisfied. During this period LOBCDER will have enough time to replicate the data according to the data owner requiements. Analysis of LOBCDER access patterns over one year shows that the time between a file was last modified and the time it is first downloaded is enough to replicate it across the backends and update all $\mathrm{R}$ masters. Figure 2 shows the Cumulative Distribution Function (CDF) of the time to replicate and download. It is clear from the CFD function that files are replicated within the first 30 seconds that follows their upload, while the probability of downloading these file within the same period is below 0.02 .

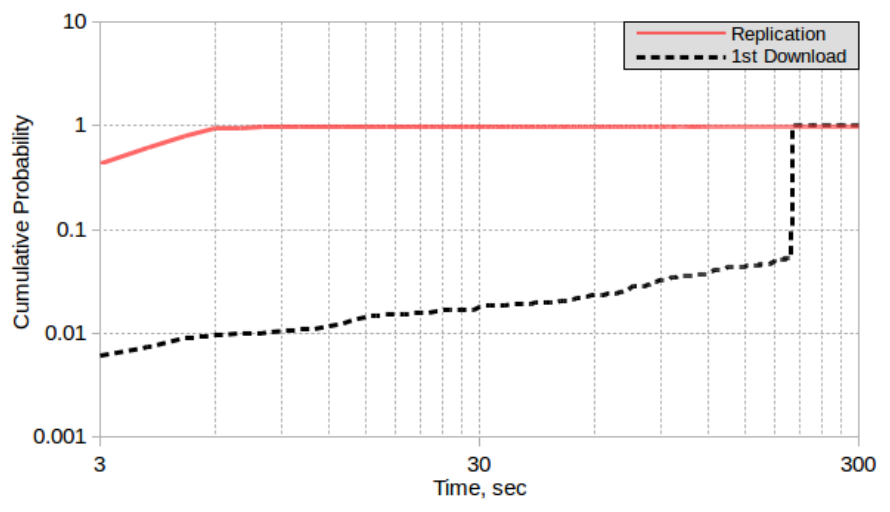

Fig. 2. Cumulative distribution function (CDF) of the time to replicate and download. The replication curve is the CDF of the time elapsed between uploading a file and replicating it. The 1st download curve is the CDF of the time elapsed between last modifying a file and downloading it for the first time.

LOBCDER achieves partition tolerance with redundancy. In our architecture we need to maintain partition tolerance on two levels: metadata and data. Partition tolerance on the metadata level is achieved by using a set of $\mathrm{R}$ masters that are constantly synchronized with the RW master. In the event the RW master becomes unavailable one of the $\mathrm{R}$ masters will replace it. Partition tolerance on the data level is achieved with data replication. 


\section{Load Balancing Algorithms}

LOBCDER offers high availability with several load balancing algorithms. To achieve this we must consider two factors: the response time and the transfer speed. To increase the transfer speed, LOBCDER must select between a number of workers based on the quality of the path (available bandwidth and actual traffic) to the data consumers.

We implemented three load-balancing algorithms. The first is a simple Round Robin (RR) algorithm which has a low response time but does not guarantee high speed data transfers. This algorithm is well suited for small data transfer typically under 10MB. With RR the RW LOBCDER master assigns incoming download requests to one worker in a cyclic fashion.

The second load balancing algorithm does not guarantee the lowest response time but can optimize data transfers. The algorithm uses Geo-location information to find the workers that is the closest to the data consumer. The GL algorithm assigns download requests to the worker which is closest to the client. This algorithm depends on a database that maps IP address to longitude and latitude coordinates. When a client requests a file, the algorithm will select the worker closest to the client. The GL algorithm is assuming that the closer the worker to the client, the better the transfer speed. The GL algorithm uses historical data from previous transfers to select between better performing workers located in the same geographical region.

With the introduction of Software Defined Networks $(\mathrm{SDN})$, more networks are becoming programmable and can provide information about their state via standardized protocols. Taking advantage of this, we developed an SDN algorithm. The SDN algorithm has a highest response time but it optimizes the speed of transfers. Therefore this algorithm is not used for small files (under $10 \mathrm{MB}$ ). When a client requests a file, the master will analyze the network load and the speed of each worker link to assign the download to the most appropriate worker. As the transfer is in progress the worker monitors the speed of the transfer and can request from the master to reroute the traffic in the case the selected path form the worker to the client is congested.

\section{Scalability ANALYSIS}

The analysis of usage data from the LOBCDER production instance on the VPH Cloud platform shows that $22 \%$ of the incoming requests involved downloads or uploads and required $93 \%$ of the total time spend serving requests. The rest of the $78 \%$ of the requests involved operations like rename and get file size and required $7 \%$ of the total time. Therefore, the major bottleneck is the transfer of files. In the VPH production platform, the LOBCDER master is able to serve more than 38,000 requests per second, which is sufficient for the current load. In case of increase of the load, multiple R masters

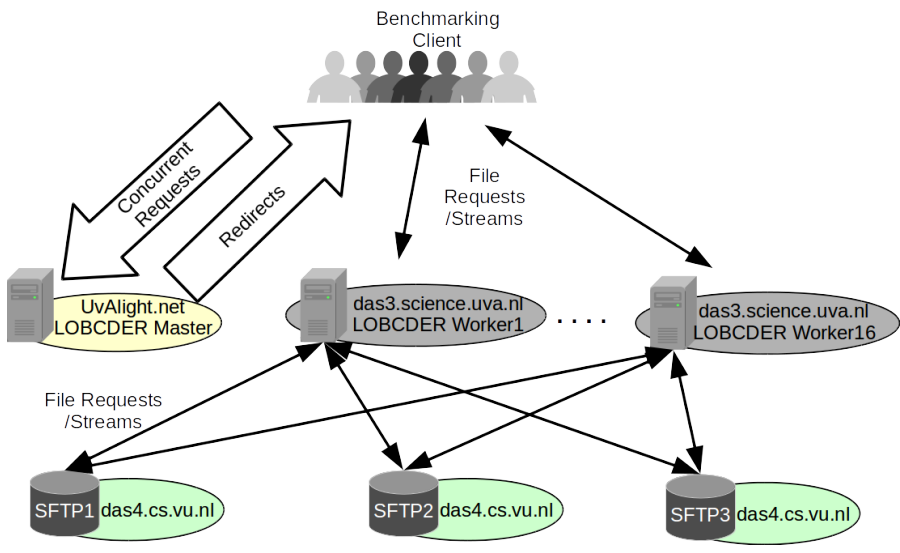

Fig. 3. Benchmark setup for testing the scalability and performance of LOBCDER. The master receives a number of concurrent request which are redirected to the workers.

can be instantiated allowing to load-balance the incoming traffic across all the masters.

To evaluate the scalability of LOBCDER in terms of file availability for concurrent requests, a set of benchmark tests where conducted. One LOBCDER RW master was deployed on a computer at the University of Amsterdam with an Intel Xeon E5450 CPU at $3.00 \mathrm{GHz}$ and $8 \mathrm{~GB}$ of memory and 16 LOBCDER workers on 16 nodes of the DAS-3 supercomputer ${ }^{5}$. Each node has a $2.4 \mathrm{GHz}$ AMD Opteron CPU and 4 GB of memory. The storage is located on three nodes hosted on the DAS-4 supercomputer and accessible through secure file transfer protocol $(\mathrm{SFTP})^{6}$. All files were replicated on all available storage and to reduce the load on the storage backend, caches were used on each LOBCDER worker. For producing concurrent requests, we used the Tsung benchmark tool ${ }^{7}$ which is able to simulate a large amount of users. Tests are composed of four separate experiments using $128,256,512$ and 1024 users requesting the same file simultaneously. On each test 1, 2, 4, 8 and 16 workers were used respectively. The benchmarking setup can be seen in Figure 3.

Figure 4 shows the average session time of all users. One session is the time between a user sends a request to download a file and the time it takes LOBCDER to complete the transfer. As the number of users increases their average wait time also increases for a fixed number of workers. This happens because each user is served by a separate thread of execution. As LOBCDER creates more threads to serve incoming requests it uses CPU and IO resources slowing down the response. When adding more workers the average waiting time of each user is drastically decreased. Adding more workers increases the rate at which users finish their download. We have measured that with 1 worker, LOBCDER serves approximately 55 users/sec and with 16 workers it serves 90

5. DAS-3:http://www.cs.vu.nl/das3/

6. DAS-4:http://www.cs.vu.nl/das4/

7. http://tsung.erlang-projects.org/ 


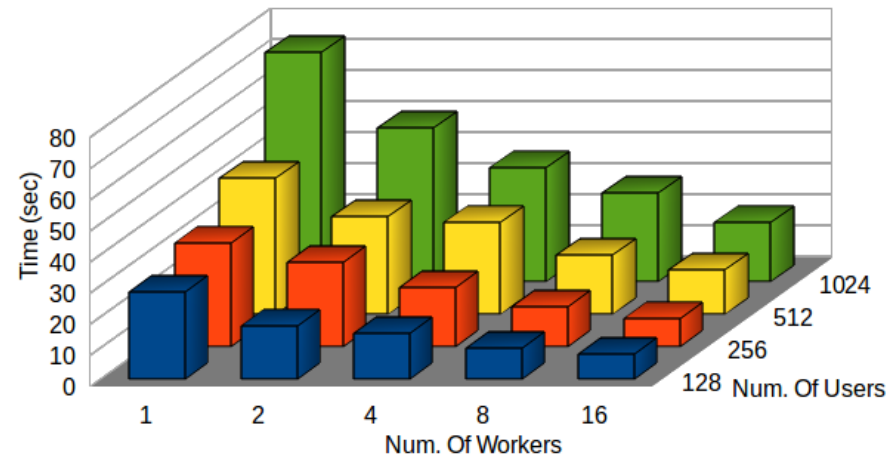

Fig. 4. Average session time of users. One session is the time between a GET request from the user and an OK response from LOBCDER

\section{users/sec}

These benchmark tests show that LOBCDER's architecture is able to cope with an increase of the number of requests and the number of users.

\section{Analysis of Cardiac Anatomy (ACA)}

In order to show how scientific workflows benefit from the proposed architecture, we present a cardiac anatomy analysis workflow. This workflow implements a novel diagnostic method to characterize the shape of the heart.

\subsection{ACA Description}

Cardiologists make therapeutic decisions based on diagnostic biomarkers extracted from the analysis of medical images. Length, diameter or wall mass of the left ventricle (LV) are universal metrics for assessing the change of the shape of the LV due to a given disease or therapeutic process. The goal of this approach is to improve the diagnosis and stratification of cardiovascular diseases through novel shape metrics based on the personalization of computational models.

The Analysis of the Cardiac Anatomy (ACA) is based on the construction of a statistical atlas of the anatomy of the LV. A statistical atlas is a description of how the shape of an organ varies within a given population. Once this atlas space is defined, each anatomy can be encoded by a set of shape coefficients: they describe the differences of each patient to the mean shape, in the axes of anatomical change defined by the modes of anatomical variation. These shape coefficients become the novel metrics to characterize shape, and guide clinical diagnosis and stratification.

The workflow, which is presented in more detail below, uses computational meshes with high order interpolation encoded by a pair of EX files ${ }^{8}$. As input, the application requires a collection of $\mathrm{LV}$ segmentations from medical images, and the main output is a set of

8. http://www.cmiss.org/cmgui/wiki/

/TheCmguiEXFormatGuideExnodeAndExelemFiles shape coefficients for each patient. In the construction of an atlas, shapes are encoded in computational meshes through an automatic personalization process from the segmented images [11], [12]. Then, a reduction of the number of shape dimensions is performed through a principal component analysis (PCA).

A cardiologist analyzes a collection of medical images which can be ultrasound, magnetic resource or computer tomography images. In a preliminary segmentation step, the anatomy of interest in each image is extracted and saved in a new image. The resulting set of segmented images is the input of the use case. The output consists of a report of the shape analysis, the computational meshes of all patients, the description of the modes of variation, and the set of shape coefficients that describe the population analyzed.

\subsection{Running ACA with LOBCDER}

In order to run the ACA workflow, a dataset consisting of medical images is first uploaded to LOBCDER. As soon as the dataset is uploaded LOBCDER starts replicating the images to the most reliable backend. A typical study is composed of about 100 images which gives a reasonable statistical significance to the results. The size of each image depends on the imaging modality and spatial resolution, ranging from $1 \mathrm{MB}$ to 65 MB. Compression of binary images heavily alleviates the data burden and users are required to aggregate the data of all patients within one single compressed folder. This also make the interaction with LOBCDER easier, as only one file needs to be transferred and the bandwidth utilization can be optimized. After uploading the compressed folder, the user accesses the interface of the anatomical model database [13] and invokes the service of calculating the atlas.

Once the image set is made available, a computational mesh is automatically fitted to each segmented anatomy [11], [12]. This process runs through a web interface to the heartgen web service [14] relaying on LOBCDER as distributed file manager to provide a shared storage space to fetch data, images, and return analysis results. Without LOBCDER, the workflow would rely on the cardiologist sending images to a researcher who would then manually set up the analysis and collect and send results back to the user. At this stage of the workflow the tasks can be executed in parallel and do not require communications. As a consequence multiple instance of the heartgen service are started to process concurrently the input datasets orchestrated by the Pumpkin framework [15]. It is installed on the VPH-cloud computing platform and uses LOBCDER as virtual file system. Pumpkin accesses the dataset needed for processing via the LOBCDER service from various computing locations leading to concurrent requests similar to the benchmark experiment described in Section 6.

Once the anatomy of each patient has been described by a computational mesh, all meshes are retrieved 
for further analysis. Because the ACA workflow uses LOBCDER as a virtual file system on the VPH-Cloud platform, all intermediate datasets generated by the workflow remain accessible regardless of geographical location and thus available for the PCA and computation of shape coefficients. The final output of the analysis is available to the user through LOBCDER.

\section{FUTURE WORK \& CONCLUSIONS}

Medical applications need access to large datasets which are geographically distributed and located in different storage infrastructures. The discovery and access of these datasets is often time-consuming for researchers that are not necessary familiar with data management services.

To address the issues of transparent, simple and efficient data access we elaborated a new method of data federation and implemented LOBCDER, a distributed data management service that is able to aggregate multiple storage types and present them to users and applications as a single storage framework. It also enables to include stateless worker instances. These workers can be deployed on available resources in order to increase data availability, request throughput and minimize session time.

Deploying LOBCDER is simple because it is implemented as a web application, allowing deployment on a variety of application servers. For an intuitive data access for users and applications LOBCDER uses a WebDAV interface.

To minimize the network overhead and the load on storage resources we will investigate cache sharing between workers. If a worker is selected to stream a large file, instead of using a storage backend, that worker pulls data from a set of available workers holding the same data in cache taking advantage of torrent-like methods. Data replication can be faster if workers replicate cached data to the closest backends.

\section{REFERENCES}

[1] S. Kavulya, J. Tan, R. Gandhi, and P. Narasimhan, "An analysis of traces from a production mapreduce cluster," in Proceedings of the 2010 10th IEEE/ACM International Conference on Cluster, Cloud and Grid Computing, ser. CCGRID '10. Washington, DC, USA: IEEE Computer Society, 2010, pp. 94-103. [Online]. Available: http://dx.doi.org/10.1109/CCGRID.2010.112

[2] I. Foster, "Globus online: Accelerating and democratizing science through cloud-based services," Internet Computing, IEEE, vol. 15, no. 3, pp. 70-73, May 2011.

[3] J. Bresnahan, M. Link, G. Khanna, Z. Imani, R. Kettimuthu, and I. Foster, "Globus GridFTP: what's new in 2007," in GridNets '07, 2007.

[4] H. Abu-Libdeh, L. Princehouse, and H. Weatherspoon, "Racs: a case for cloud storage diversity," in Proceedings of the 1st ACM symposium on Cloud computing, ser. SoCC ' 10 . New York, NY, USA: ACM, 2010, pp. 229-240. [Online]. Available: http://doi.acm.org/10.1145/1807128.1807165

[5] D. Testi, P. Quadrani, and M. Viceconti, "PhysiomeSpace: digital library service for biomedical data," Physical and Engineering Sciences, vol. 368, no. 1921, pp. 2853-2861, Jun. 2010. [Online]. Available: http://dx.doi.org/10.1098/rsta.2010.0023

[6] A. Rajasekar, M. Wan, R. Moore, and W. Schroeder, "A prototype rule-based distributed data management system," in HPDC workshop on Next Generation Distributed Data Management, 2006.
[7] Ł. Dutka, R. Słota, M. Wrzeszcz, D. Król, and J. Kitowski, “Uniform and efficient access to data in organizationally distributed environments," in eScience on Distributed Computing Infrastructure. Springer, 2014, pp. 178-194.

[8] S. Benkner, C. Borckholder, M. Bubak, Y. Kaniovskyi, R. Knight, M. Koehler, S. Koulouzis, P. Nowakowski, and S. Wood, "A cloudbased framework for collaborative data management in the vphshare project," in Advanced Information Networking and Applications Workshops (WAINA), 2013 27th International Conference on. IEEE, 2013, pp. 1203-1210.

[9] S. Koulouzis, D. Vasyunin, R. Cushing, A. Belloum, and M. Bubak, "Cloud data federation for scientific applications." in Euro-Par Workshops, 2013, pp. 13-22.

[10] S. Gilbert and N. Lynch, "Brewer's conjecture and the feasibility of consistent, available, partition-tolerant web services," SIGACT News, vol. 33, no. 2, pp. 51-59, Jun. 2002. [Online]. Available: http://doi.acm.org/10.1145/564585.564601

[11] P. Lamata, M. Sinclair, E. Kerfoot, A. Lee, A. Crozier, B. Blazevic, S. Land, A. J. Lewandowski, D. Barber, S. Niederer, and N. Smith, "An automatic service for the personalization of ventricular cardiac meshes," Journal of The Royal Society Interface, vol. 11, no. 91, 2014. [Online]. Available: http://rsif. royalsocietypublishing.org/content/11/91/20131023.abstract

[12] P. Lamata, S. Niederer, D. Nordsletten, D. C. Barber, I. Roy, D. Hose, and N. Smith, "An accurate, fast and robust method to generate patient-specific cubic hermite meshes," Medical image analysis, vol. 15, no. 6, pp. 801-813, 2011.

[13] D. Gianni, S. McKeever, and N. Smith, "euheartdb: a web-enabled database for geometrical models of the heart," in Functional Imaging and Modeling of the Heart. Springer, 2009, pp. 407-416.

[14] E. Kerfoot, P. Lamata, S. Niederer, R. Hose, J. Spaan, and N. Smith, "Share and enjoy: anatomical models databasegenerating and sharing cardiovascular model data using web services," Medical $\mathcal{E}$ biological engineering $\mathcal{E}$ computing, vol. 51, no. 11, pp. 1181-1190, 2013.

[15] R. Cushing, A. Belloum, M. Bubak, and C. D. Laat, "Automatabased dynamic data processing for clouds," in Euro-Par 2014: Parallel Processing Workshops, 2014. 